\title{
Epilepsy-associated neurocognitive disorders (EAND) in an onchocerciasis-endemic rural community in Cameroon:
}

\section{A population-based case-control study}

\author{
Alfred K. Njamnshi a,b,c,*, Eric-Samuel Chokote ${ }^{\mathrm{a}, \mathrm{b}, \mathrm{c}}$, Leonard Ngarka ${ }^{\mathrm{a}, \mathrm{b}, \mathrm{c}}$, Leonard N. Nfor ${ }^{\mathrm{a}, \mathrm{b}, \mathrm{c}}$, \\ Earnest N. Tabah ${ }^{\mathrm{a}, \mathrm{c}, \mathrm{d}}$, Jonas G. Basseguin Atchou ${ }^{\mathrm{a}, \mathrm{b}, \mathrm{c}}$, Samuel A. Angwafor ${ }^{\mathrm{b}, \mathrm{e}, \mathrm{h}}$, Cyrille Nkouonlack ${ }^{\mathrm{b}, \mathrm{f}, \mathrm{g}}$, \\ Michel K. Mengnjo ${ }^{\mathrm{a}, \mathrm{b}, \mathrm{c}}$, Wepnyu Y. Njamnshi ${ }^{\mathrm{b}, \mathrm{c}, \mathrm{i}}$, Fidèle Dema ${ }^{\mathrm{a}, \mathrm{b}, \mathrm{d}}$, Godwin Y. Tatah ${ }^{\mathrm{a}, \mathrm{b}, \mathrm{j}}$, \\ Anne-Cecile Zoung-KanyiBissek ${ }^{\mathrm{c}, \mathrm{k}}$, Jean-Marie Annoni ${ }^{\mathrm{b}, \mathrm{i}}$, Nicolas Ruffieux ${ }^{\mathrm{l}}$ \\ a Department of Neurology, Central Hospital Yaoundé, Yaoundé, Cameroon \\ ${ }^{\mathrm{b}}$ Brain Research Africa Initiative (BRAIN), Yaoundé, Cameroon E' Geneva, Switzerland \\ ' Faculty of Medicine E' Biomedical Sciences, The University of Yaoundé I, Yaoundé, Cameroon \\ ${ }^{\mathrm{d}}$ Ministry of Public Health, Yaoundé, Cameroon \\ e Department of Neurology, University College London, London, United Kingdom \\ ${ }^{\mathrm{f}}$ Department of Neurology, Regional Hospital Buea, Buea, Cameroon \\ ${ }^{g}$ Department of Clinical Sciences, Faculty of Health Sciences, University of Buea, Buea, Cameroon

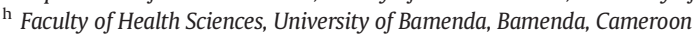 \\ i Department of Neurology and Neuropsychology, University of Fribourg, Fribourg, Switzerland \\ ${ }^{j}$ Neurology Department, CH Saint-Nazaire, Saint-Nazaire, France. \\ ${ }^{k}$ Department of Health Operations Research, Ministry of Public Health, Yaoundé, Cameroon \\ ${ }^{1}$ Department of Special Education, University of Fribourg, Fribourg, Switzerland
}

Keywords:

Epilepsy

Cognitive impairment

Executive function

Verbal fluency

Rural population

Cameroon

\begin{abstract}
A B S T R A C T
Background: Epilepsy affects at least 50 million individuals worldwide, especially in sub-Saharan Africa (sSA). Cognitive impairment is common in people with epilepsy (PWE) yet, little is known on the burden of cognitive impairment in people with epilepsy in sSA. This study was thus designed to assess cognitive impairment in PWE or epilepsy-associated neurocognitive disorders (EAND) in a rural population in Cameroon.

Methods: This was a case-control study including PWE and age/sex-matched healthy controls from July to September 2017 in Bilomo, a village in the Mbam and Kim Division. The Montreal Cognitive Assessment (MoCA), International HIV Dementia Scale (IHDS), Dubois' Five Word testing, Frontal Assessment Battery (FAB), Isaac's Set Test and the Clock drawing test were administered to the study participants to evaluate global and specific cognitive functions.

Results: Eighty participants were included ( 40 cases and 40 controls) with a mean age of 25.78 years. Using the MoCA, 87.5\% of cases had cognitive impairment, against $37.5 \%$ of controls ( $<<0.001$; OR 11.67; CI 3.4045.09). Using the IHDS, the prevalence of global cognitive impairment was $84.6 \%$ among the cases against $40 \%$ for the controls ( $\mathrm{p}=<0.001$; OR 7.07; CI 2.29-29.19). Specifically, executive function deficits $(92.5 \%$ of cases vs $40.0 \%$ of controls $\mathrm{p}=<0.001 \mathrm{OR}=18.50 \mathrm{CI} ; 4.48-105.08$ ) and decreased verbal fluency ( $100 \%$ of cases against $45 \%$ of controls $\mathrm{p}<0.001$ ) were the most affected cognitive domains. Longer duration of epilepsy and higher seizure frequency were associated with global cognitive impairment. Low level of education was associated with both decreased verbal fluency and executive dysfunction while a longer stay in Bilomo correlated with poor results on the Isaac's Set Test.

Conclusion: The prevalence of cognitive impairment appears to be much higher in PWE in the Mbam valley, particularly decreased executive function and verbal fluency, than in people without epilepsy. Longer disease duration, higher seizure frequency, low level of education and length of stay in Bilomo are associated with poorer cognitive performance. More studies are needed to refine evaluation tools to better characterize and manage EAND in sSA.
\end{abstract}

\footnotetext{
* Corresponding author at: Neuroscience Laboratory, Faculty of Medicine, The University of Yaoundé I, Brain Research Africa Initiative (BRAIN), PO Box 25625, Yaoundé, Cameroon. E-mail address: alfred.njamnshi@brainafrica.org (A.K. Njamnshi).
} 


\section{Introduction}

Epilepsy is one of the most common neurological diseases, affecting more than 50 million individuals worldwide [1,2]. The burden of epilepsy is particularly important in sub-Saharan Africa ( $\mathrm{sSA}$ ) where the prevalence is between 2.2 and 58 per 1000 [3]. The values of prevalence of epilepsy in some parts of Cameroon are among the highest in the world, ranging from 11.6 to 134 per 1000 [4]. The causes and risk factors for this high prevalence of epilepsy in this and other parts of Cameroon are not fully understood but there is a growing body of evidence suggesting that onchocerciasis may be $[5,6]$. Onchocerciasis is a parasitic infection present mainly in Africa, particularly in regions with fast flowing rivers with constitute a favorable breeding ground for the nematode Onchocerca volvulus. It has been known classically to have mainly ocular and dermatological manifestations; however other systemic involvements have been described [7] and brain involvement in increasingly being recognized. Two recent population-based prospective studies in the Mbam and Sanaga valleys in Cameroon have demonstrated a strong association between onchocerciasis and epilepsy with a dose effect and temporal relationship $[8,9]$. In this region, the mortality ratio is 2-3 times higher in people with epilepsy (PWE) compared to the general population with deaths attributed either to the cause of epilepsy or to the epilepsy itself mostly related to sudden unexplained death in epilepsy (SUDEP) and status epilepticus [10]. The rapidly growing body of evidence suggesting that onchocerciasis induces epilepsy has led to the concept of onchocerciasis-associated epilepsy (OAE) $[5,6]$. The exact pathophysiological mechanism of OAE is not well understood but an auto-immune mechanism [11] and direct or indirect effect of the microfilaria on the CNS has been proposed [8].

The burden of OAE warrants urgent action to provide care for the PWE and in many cases, their families who are often stigmatized and left with little or no access to care [12].

Most of the focus of clinical care in epilepsy is directed at controlling seizures, neglecting its other medical, psychological and socio-economic complications which contribute largely to its morbidity [10]. Neurocognitive disorders are increasingly being recognized as frequent and disabling complications of epilepsy. The frequency of cognitive impairment in epilepsy ranges between 20 and 72\% [2,3,13]. Various cognitive domains can be affected including: verbal and visual memory, orientation, constructional praxis, language, executive functions, attention and psychomotor speed [3,14]. These defects have multiple causes, such as structural brain lesions, the seizures (and the secondary lesions they induce), and antiepileptic drugs [15]. The consequences of cognitive deficiencies are significant, resulting in poor academic performance and school dropouts, unemployment and poor medication adherence, all of which reduce the quality and life expectancy of affected individuals [16].

Given the paucity of data on cognitive impairment in people with epilepsy in SSA and Cameroon in particular, this study was designed to assess epilepsy-associated neurocognitive disorders in a rural Cameroonian population.

\section{Participants and Methods}

\subsection{Study site, design and participants}

This was a community-based, case-control study which included people with epilepsy and healthy controls both residing in Bilomo. Bilomo is a village situated $120 \mathrm{Km}$ north of Yaoundé in the Mbam and Kim division in the Centre region of Cameroon (See Fig. 1). Its population is made of the Sanaga people, a sub-ethnic group of the Bantous. In this region, the Sanaga language is the most spoken language and French is the more commonly used of the two official languages of Cameroon. The geographical features of this region with savanna vegetation in close proximity with the dense and humid equatorial forest, temperatures varying between 22 and $25{ }^{\circ} \mathrm{C}$ and annual rainfall between 1500 and $2000 \mathrm{~mm}$, have rendered it an endemic focus for many parasitic diseases such as onchocerciasis. Onchocerciasis is endemic in this area and the inhabitants of the village who are mostly

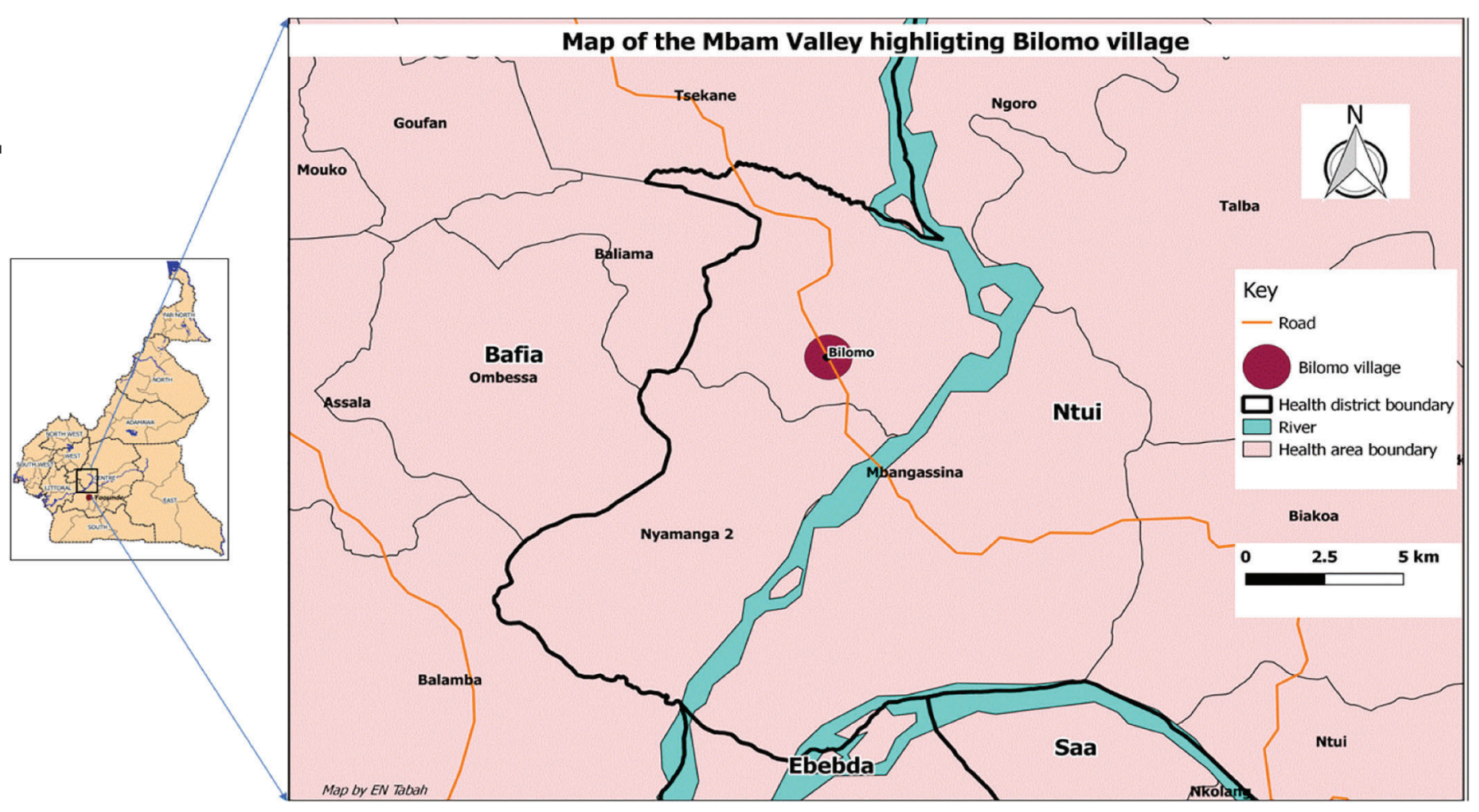

Fig. 1. Map showing study site. 
peasant farmers have been receiving ivermectin mass treatment since 1991. Previous studies have reported a particularly high prevalence of epilepsy in the Mbam valley and in Bilomo in particular [17]. In 2017, the total population of Bilomo was 1321 with a crude epilepsy prevalence of $4.6 \%$ [18]. The current study was a follow-up of this epidemiological study.

Participants were recruited between July and September 2017 as a follow-up of a larger door-to-door study in the area. Cases were persons presenting with active epilepsy (defined as: at least two unprovoked seizures within a year, with at least one in the past five years independent of epileptic treatment status), aged 8 years and above and residing in Bilomo for at least 6 months. The cut-off of 8 years was chosen because of the age limit imposed by the screening tests used in our study. Controls were age $(+/-2)$ and sex-matched healthy and epilepsy-free individuals, living in the same village for at least six months. Informed consent was obtained from all adult participants. For participants less than 18 years, assent was obtained from them and consent from their parents or guardians. Acutely ill individuals and those unable to give accurate information on their medical history or undergo cognitive testing were excluded from the study. All participants who could not understand or speak French were equally excluded.

\subsection{Procedure}

After obtaining authorizations from the competent administrative and health authorities, a door-to-door survey was carried out in the village of Bilomo. This survey was done with the help of the village chief, quarter heads and local community health workers who provided a detailed description of the village and accompanied the research team to facilitate access to the different households. In each household, the family head was interviewed by a physician with experience in neuroepidemiological surveys, to identify all people likely to have epilepsy in the household. All the suspected cases of epilepsy were further evaluated by a Neurologist or another physician with epilepsy training. Participant data collected included: identification code, the history of epilepsy and other contributive past medical history and results of the physical examination especially the neurological assessment. A general physical examination was also carried out with particular emphasis on the skin and the musculoskeletal system in search of active lesions of onchocerciasis and also scars, burns and fractures often seen as consequences of epileptic seizures. All participants with peculiar skin lesions where reviewed by a dermatologist (ACZK). Neuropsychological evaluation was done by a neurologist trained in neuropsychological testing (SEC, LN, MKM) with the following cognitive screening tests: the Montreal Cognitive Assessment (MoCA), the International HIV Dementia Scale (IHDS), the Dubois five-word testing, the Frontal Assessment Battery (FAB), the Isaac's set test and the clock drawing test.

Control participants were conveniently selected in Bilomo either from the same household or from neighboring households where PWE were identified. All potential controls were assessed by a Neurologist to ensure that none had epilepsy or a history of seizures. Those who met the inclusion criteria and who signed the informed consent forms were matched by age and sex with the already enrolled cases. Neuropsychological evaluation was same in controls as that done in cases. All interviews were conducted in French which is widely spoken in region.

In the earlier epidemiological study which preceded this phase, the SD Bioline Onchocerciasis Ov16 IgG4 rapid diagnostic test (RDT) (Standard Diagnostics, Gyeonggi-do, South Korea) for anti-Onchocerca antibodies had been carried out in all participants with epilepsy and in children between 7 and 10 years in order to assess the ongoing onchocerciasis transmission in the village [18].

\subsection{Study Instruments}

> The Montreal Cognitive Assessment (MoCA) is a composite battery used to evaluate global cognitive impairment. Cognitive domains assessed are memory, language, orientation, visuospatial functions, executive functions and attention. It is an 8-Item questionnaire with a total score of 30 . Individuals with less than 12 years of education, score an additional point and a score less than 26 is considered pathologic. This cut-off was determined by comparing the results of patients with either mild cognitive impairment or mild Alzheimer's disease (MMSE $\geq 27$ ) against 90 healthy volunteers recruited in Canada [19]. It was chosen over the conventional MMSE because of its proven better sensitivity to detect cognitive defects in epilepsy patients in Thailand [20]. Several authors have proposed to revise the cut-off according to the level of education of the population considered. A recent study which evaluated the accuracy of a Ki-swahili version of the MoCA in a rural African population proposed an optimal cut-off of 19 to detect Mild cognitive impairment. This was adopted in our study considering the sociodemographic characteristics of our study sample [21] and this is the cut-off we used in this study. The MoCA test has equally been validated for use in children and adolescents between 14 and 21 years old [22].

> The International HIV Dementia Scale (IHDS) is a 3-item test used as a screening instrument for global cognitive dysfunction in the HIV infected individuals [23]. It focuses on memory, motor and psychomotor speed and lasts about $5 \mathrm{~min}$. It is scored on 12 and a result $\leq 10$ defines cognitive impairment. This test was developed after evaluation of two cohorts of HIV positive individuals in the USA and in Uganda and was thus proven useful in both industrialized and developing countries [23]. It was used for this study because of its ease of administration and effectiveness in measuring cognitive performance in Cameroon [24].

> The Dubois' five-word testing is specific for verbal episodic memory impairment. It is scored on 10 and a score of less than 9 is considered pathologic [25]. This cut off was determined after comparing the results of 86 patients with Alzheimer's disease against 126 participants complaining of functional memory disorders in France [25]. It has been validated mostly for neurodegenerative diseases [26]. Its use has been suggested in epilepsy patients particularly in temporal lobe epilepsy [27]. Though initially designed in an industrialized setting, a few studies employed the Dubois' five-word testing in rural African populations. It was used as a screening test for Alzheimer's dementia in a large study conducted in three countries of Central and West Africa (Benin, The Republic of Congo and The Central African Republic). This study included 2657 individuals, 57\% of whom did not have a formal education. The authors however, maintained the cut-off proposed by Dubois et al. $[28,29]$. A Tunisian research group concluded that the standard cut-off of $9 / 10$ could be sensitive enough in a population of young Tunisian adults $(<50$ years) who had not completed their primary education. In this study the five words were however translated into Arab [30]. The Dubois' five-word testing was the only test used in our study which required the ability to read.

> The Frontal Assessment Battery (FAB) assesses executive functions and attention and is frequently used in clinical practice [31]. It evaluates 6 items each scored on three with a total of 18 . A score less than 16 is considered pathologic for a literate participant and less than 15 for the uneducated. This bedside battery was developed by comparing 121 subjects with various neurodegenerative diseases resulting in frontal lobe dysfunction against 42 normal French subjects [31].We adopted a cut-off of 13 in conformity with recommendations from a Brazilian study which adjusted the cut-off to the level of education of normal healthy individuals [32]. Moreover, it has been shown that the FAB is a short and simple-to-use clinical test which is sensitive enough to diagnose executive dysfunction in patients with Temporal Lobe Epilepsy (TLE) [33]. The FAB has been previously used in rural populations in Africa, particularly in Madagascar where it was employed to assess executive dysfunctions in patients who had suffered a stroke [34]. The FAB has also been used to evaluate executive cognitive function in Brazilian 
adolescents (mean age 14 years) who suffered maltreatment and were substance abusers. In this case-control study, the participants equally had a low level of education [35].

- Isaac's Set Test was used to evaluate verbal fluency. It is scored on 40 with a cut off of 30 defining a pathologic condition [36]. It was originally developed by testing 189 elderly subjects in Glasgow with some degree of mental disease requiring assistance in daily living activities [37]. Corne et al. in 2012 used the Isaac's Set Test for presurgical neuropsychological evaluation of patients with TLE [38]. It was also used in the EDAC (Epidemiology of Dementia in Central Africa) survey to evaluate verbal fluency in the Central African Republic and the Republic of Congo [29].

> The clock drawing test (CDT) was employed to assess visuospatial functions. It evaluates the participant's ability to represent a specific time on a well-drawn clock. The accuracy of the contours, numbering and hand placements to indicate 10 min past 11 were graded on 6 such that a score $\geq 3$ was considered abnormal [39]. The test was developed in the USA by comparing the results of 46 patients diagnosed with clinically probable Alzheimer dementia with those of 26 normal elderly controls [39]. The CDT has been validated as a screening test for cognitive impairment in epilepsy patients [40]. Its utility has also been shown in rural populations in Portugal [41]. The clock drawing test has been shown to be sensitive enough to diagnose cognitive impairment in children and adolescents between 8 and 14 years old with Attention Deficit Hyperactivity Disorder (ADHD) compared to healthy controls, in a study in Iran [42].

\subsection{Seizure and epilepsy classification}

Seizures were described as either focal onset, generalized onset, focal onset evolving to bilateral tonic clonic seizures or unclassified seizures - according to the 2017 classification of the ILAE [43]. To these categories was added nodding seizures, a specific seizure type described in onchocerciasis associated epilepsy [44]. However, the 1989 classification of epilepsy (etiologies) [45] was preferred considering the absence of genetic and imaging studies indispensable in the 2017 ILAE classification. Symptomatic epilepsy was considered in participants who had a history of perinatal, infectious or traumatic lesions prior to the onset of the first seizure. Those who had focal seizures and in whom no obvious cause could be found were labeled as cryptogenic epilepsy. When the seizures where described as generalized and no obvious cognitive impairment found, the participant was categorized as idiopathic epilepsy and those who could not fit in any of these where labeled unknown. Considering the high prevalence of onchocerciasis in this region, onchocerciasisassociated epilepsy (OAE) was included in the etiological classification following the criteria proposed by Colebunders et al. [46]. These include:

1) Person living for at least 3 years in an O. volvulus-endemic region;

2) High prevalence of epilepsy in the village and families having more than one child with epilepsy;

3) History of at least two unprovoked epileptic seizures $24 \mathrm{~h}$ apart;

4) No other obvious cause for the epilepsy (mainly established by good history taking to exclude common causes such as perinatal, infectious or traumatic);

5) Onset of epilepsy between the ages of 3 and 18 years;

6) Normal psycho-motor development before the onset of epilepsy.

\subsection{Data analysis}

Data was analyzed using SPSS version 20.0. Pearson's Chi squared test was used for comparisons between categorical data. Means of continuous variables were compared between groups with the aid of the one-way multiple analyses of variance (1-ANOVA).

Bivariate and multivariate analyses (logistic regression) were performed to determine which factors which were associated with global and specific cognitive dysfunctions. Factors tested included: age, gender, education level, length of stay in the village, frequency of seizures, duration of epilepsy, use of antiepileptic drugs and adherence to epilepsy treatment. All statistics tests were two-sided and considered statistically significant at $\mathrm{p}<0.05$.

\section{Results}

3.1. Socio-demographic data and characteristics of epilepsy in the study sample

Out of a total population of 1321 inhabitants, 1271 individuals were screened for epilepsy during the door-to-door survey. Of the 60 suspected cases of epilepsy, 57 were found to have epilepsy. The details of this survey are reported elsewhere $[6,16,35]$. Forty-four of the fifty-seven eligible PWE were enrolled in the study together with corresponding 44 controls. Four cases and four controls had incomplete data and were

Table 1

General characteristics of the study population.

\begin{tabular}{|c|c|c|c|c|c|c|}
\hline \multirow[t]{2}{*}{ Variable } & \multirow[t]{2}{*}{ Category } & \multicolumn{2}{|l|}{ PWE } & \multicolumn{2}{|l|}{ Controls } & \multirow[t]{2}{*}{ P-value } \\
\hline & & $\mathrm{N}$ & $\%$ & $\mathrm{~N}$ & $\%$ & \\
\hline Total & Total & 40 & $100.0 \%$ & 40 & $100.0 \%$ & \\
\hline Mean age & Year \pm SD & $25.90 \pm 8.35$ & & $25.65 \pm 9.28$ & & 0.9 \\
\hline Mean length of stay in Bilomo & Year \pm SD & $23.63 \pm 10.32$ & & $18.40 \pm 11.95$ & & 0.04 \\
\hline \multirow[t]{6}{*}{ Occupation } & Civil Servant & 0 & $0.0 \%$ & 1 & $2.5 \%$ & \\
\hline & Formal Private & 0 & $0.0 \%$ & 1 & $2.5 \%$ & \\
\hline & Informal Private & 1 & $2.5 \%$ & 2 & $5.0 \%$ & \\
\hline & Agriculture & 29 & $72.5 \%$ & 21 & $52.5 \%$ & 0.065 \\
\hline & Unemployed & 6 & $15.0 \%$ & 2 & $5.0 \%$ & \\
\hline & Student/Pupil & 4 & $10.0 \%$ & 13 & $32.5 \%$ & \\
\hline \multirow[t]{4}{*}{ Tribe } & Mbamois & 38 & $95.0 \%$ & 36 & $90.0 \%$ & 0.708 \\
\hline & Bamileke & 0 & $0.0 \%$ & 1 & $2.5 \%$ & \\
\hline & Foulani & 1 & $2.5 \%$ & 2 & $5.0 \%$ & \\
\hline & Others & 1 & $2.5 \%$ & 1 & $2.5 \%$ & \\
\hline \multirow[t]{3}{*}{ Level of education } & None & 2 & $5.0 \%$ & 0 & $0.0 \%$ & \\
\hline & Primary & 36 & $90.0 \%$ & 21 & $52.5 \%$ & \\
\hline & Secondary & 2 & $5.0 \%$ & 19 & $47.5 \%$ & $<0.001$ \\
\hline \multirow[t]{2}{*}{ Gender } & Female & 26 & $65.0 \%$ & 26 & $65.0 \%$ & 1.000 \\
\hline & Male & 14 & $35.0 \%$ & 14 & $35.0 \%$ & \\
\hline \multirow[t]{4}{*}{ Marital_Status } & Single & 28 & $70.0 \%$ & 24 & $60.0 \%$ & \\
\hline & Married & 7 & $17.5 \%$ & 14 & $35.0 \%$ & 0.0218 \\
\hline & Widowed & 1 & $2.5 \%$ & 1 & $2.5 \%$ & \\
\hline & Divorced & 4 & $10.0 \%$ & 1 & $2.5 \%$ & \\
\hline
\end{tabular}


excluded from analysis. The total of study sample population was therefore 80: 40 cases (PWE +) and 40 age and sex-matched controls (PWE-).

The general characteristics of the sample population are summarized in Table 1. The mean age in the total sample was $25.78 \pm 8.77$ years with close to $20 \%$ of the study sample aged less than 18 . There was a female predominance in the study sample with 52 (65\%) females against 28 males (35\%) with a female/male sex ratio of 1.9 . Seventyeight (97.5\%) participants had been to school, but fewer cases made it to secondary school (5.0\% of PWE + against $47.5 \%$ of controls; p < 0.001 ). The mean length of stay in the village was higher for the cases than the controls. (See Table 1.)

The mean age at the first seizure was $12.15 \pm 4.14$. The most frequent type of seizures was generalized (mostly generalized tonic-clonic and absence seizures), representing $82.5 \%$ of seizure types (See Table 2 ). Head nodding was reported in 2 participants (5\%). More than half of the cases had at least one seizure a week while $46 \%$ had had a seizure a few days prior to the interview. About two-thirds of PWE were on antiepileptic treatment, mostly carbamazepine (55.5\%) and phenobarbital (48.1\%). About one quarter of PWE admitted non-adherence to the antiepileptic drug (AED).

The OV16IgG4 antibodies were detected in $32.5 \%$ (13/40) of the case group.

\subsection{Neuropsychological assessment}

\subsubsection{Global cognitive impairment}

The neuropsychological performance of the sample population is presented in Tables 3 to 5 .

The Montreal Cognitive Assessment (MoCA) and the International HIV-associated Dementia Scale (IHDS) were used to evaluate global cognitive function. Mean scores were significantly lower in PWE compared to the control group. The proportion of participants with pathologic MoCA scores when considering the standard cut-off $(<26)$ did not differ significantly between cases and controls. However, with the cut-off at 19, the difference was statistically significant. (see Tables 3, 4 and 5). The

Table 2

Characteristics of Epilepsy.

\begin{tabular}{llll}
\hline Variable & Category & $\mathrm{N}$ & $\%$ \\
\hline \multirow{3}{*}{ Seizure type } & Total & 40 & $100.0 \%$ \\
& Generalized & 33 & $82.5 \%$ \\
& Focal evolving to bilateral tonic-clonic & 3 & $7.5 \%$ \\
& Unclassified & 2 & $5.0 \%$ \\
Epilepsy etiology & Nodding Seizures & 2 & $5.0 \%$ \\
& Idiopathic & 1 & $2.5 \%$ \\
& Symptomatic & 0 & $0 \%$ \\
& Cryptogenic & 21 & $52.5 \%$ \\
& Unknown & 16 & $40.0 \%$ \\
& Nodding Seizures & 2 & $5.0 \%$ \\
& OAE & 38 & $95 \%$ \\
& More than once week & 9 & $22.5 \%$ \\
& Once a week & 12 & $30.0 \%$ \\
& More than once a month & 5 & $12.5 \%$ \\
& Once a month & 6 & $15.0 \%$ \\
& Once every 3 months & 4 & $10.0 \%$ \\
& Once every 6 months & 2 & $5.0 \%$ \\
Duration AED use & Once a year & 1 & $2.5 \%$ \\
& Less than a year & 1 & $2.5 \%$ \\
& 1-5 years & 7 & $17.5 \%$ \\
& Greater than 5 years & 19 & $47.5 \%$ \\
& Not on AED & 13 & $32.5 \%$ \\
& Benzodiazepines, Phenobarbital & 1 & $3.7 \%$ \\
& Carbamazepine & 15 & $55.6 \%$ \\
& Phenobarbital & 11 & $40.7 \%$ \\
& No & 6 & $25.0 \%$ \\
& Yes & 18 & $75.0 \%$ \\
\hline
\end{tabular}

\footnotetext{
* Only 27 PWE were on AED.

** Information on adherence to AED could be gotten only from 24 of the 27 PWE on antiepileptic drugs.
}

prevalence of cognitive impairment based on the IHDS was $84.6 \%$ among people with epilepsy and $40.0 \%$ in healthy subjects ( $\mathrm{p}=$ $<0.001$; OR 3.37; CI 1.60-7.07).

3.2.2. Results of Neuropsychological tests evaluating specific cognitive functions

The scores on the Frontal Assessment Battery (FAB) which assessed executive functions were significantly lower in the PWE than controls. Verbal fluency scores on the Isaac's Set test were equally much lower in the PWE group; all participants with epilepsy had a pathologic score $(p<0.001)$. Learning and memory scores (Dubois' five-word testing) were comparable in both the cases and controls.

The mean scores on the clock test were better in the control group; $3.7 \pm 1.76$ vs $4.67 \pm 1.38$ for the controls and cases respectively ( $\mathrm{p}=$ 0.008 ). However, there was no difference between both groups in the proportion of participants with pathologic responses.

\subsubsection{Neuropsychological parameters for Cases and Controls with Primary School education level only}

In order to adjust for the difference in educational level between cases and controls, the results of the test scores were compared in a sub-population of PWE + and PWE- who attended only primary school (see Table 6).

The proportion of cases with pathologic scores was significantly higher for the IHDS, the FAB, and the Isaac's set test. Learning and memory scores as well as visuospatial functions evaluated by the clock test where comparable in both sub-groups.

\subsubsection{Factors Associated with Cognitive impairment}

Following a stepwise backward likelihood binary logistic regression of each neurocognitive test and inputting all potential variables likely to have an influence on the test performance, the frequency of seizures was the only independently associated factor with poor IHDS scores in PWE (OR 2.252; $p=0.055$ ). Length of stay in the village and level of education were the two factors associated with decreased verbal fluency on the Isaac's set test (see Table 7). Those who had stayed for more than 15 years in Bilomo had an OR of 1.094 for decreased verbal fluency. The level of education was equally associated with executive dysfunction as the odds of having a poor result on the FAB were 8.96 for participants who had attended only primary school.

There was a significant negative correlation on the Spearman's correlation test between duration of epilepsy and global cognitive performance both with the MoCA $(r=-0.139, p=0.048)$ and $\operatorname{IHDS}(r=-0.342 \mathrm{p}=$ 0.036).

\section{Discussion}

Cognitive impairment is a frequently encountered but neglected problem in epilepsy $[2,3,10,13]$. To the best of our knowledge, this is the first study to systematically assess cognitive function of PWE in an onchocerciasis-endemic area in sub-Saharan Africa. The prevalence of cognitive impairment in cases (84.6\%) and controls (40\%) is higher than most values of prevalence reported in literature. The prevalence of cognitive impairment in the adult population in SSA is estimated at 6.3\% [47]. Studies in South Eastern Nigeria in 2016 [3] and in Taiwan in 2005 [48] reported prevalence values of $19.6 \%$ and $36 \%$ respectively of cognitive impairment in PWE. Another study of cognitive impairment in sub group of epilepsy patients living in an onchocerciasis endemic region in Democratic republic of Congo and specifically presenting nodding seizures revealed a prevalence of $71.9 \%$ [49]. In this study however, cognitive functions were not studied with a standard neuropsychological battery. Similarly in Germany, it was reported that $72.1 \%$ of PWE had executive dysfunction and memory impairment [13].

Comparison of the prevalence of cognitive impairment in people with epilepsy between studies must take into account differences in study design especially cognitive tests used, cognitive functions assessed, and 
Table 3

Comparison of mean scores of neurocognitive tests between PWE and controls.

\begin{tabular}{|c|c|c|c|c|}
\hline $\begin{array}{l}\text { Neurocognitive test } \\
\text { (Normal cut-off) }\end{array}$ & Participants & $\mathrm{N}$ & Mean & P-value (t-test for equality of means) \\
\hline \multirow[t]{2}{*}{ MOCA ( $\geq 19)$} & PWE & 40 & $13.00 \pm 5.85$ & $<0.001$ \\
\hline & Controls & 40 & $20.23 \pm 4.94$ & \\
\hline \multirow[t]{2}{*}{ FAB $(\geq 13)$} & PWE & 40 & $6.83 \pm 3.88$ & $<0.001$ \\
\hline & Controls & 40 & $13.05 \pm 4.38$ & \\
\hline \multirow[t]{2}{*}{ Dubois $(\geq 9)$} & PWE & 40 & $7.88 \pm 2.40$ & 0.163 \\
\hline & Controls & 40 & $8.58 \pm 2.04$ & \\
\hline \multirow[t]{2}{*}{ Isaac $(\geq 30)$} & PWE & 39 & $14.59 \pm 5.67$ & $<0.001$ \\
\hline & Controls & 40 & $28.60 \pm 8.35$ & \\
\hline \multirow[t]{2}{*}{ CDT $(<3)$} & PWE & 39 & $4.67 \pm 1.38$ & 0.008 \\
\hline & Controls & 40 & $3.70 \pm 1.76$ & \\
\hline \multirow[t]{2}{*}{ IHDS $(\geq 11)$} & PWE & 39 & $7.31 \pm 3.05$ & $<0.001$ \\
\hline & Controls & 40 & $10.18 \pm 2.07$ & \\
\hline
\end{tabular}

subgroups of epilepsy patients included. Comparing data across different settings would therefore require standardization of cognitive evaluation in epilepsy patients as is the case with certain neurodegenerative diseases [50]. The prevalence reported in our study might be overestimated owing to the limitations of the tests used. Most of these tests have not been validated and may not be culturally adapted in LMICs especially in a rural population like ours [51,52]. For example, in the MoCA test, the naming of rhinoceros picture was not adapted to this population, as this animal is very unfamiliar to the inhabitants of this rural area. In South Africa, the MoCA was compared with the Repeatable Battery for the Assessment of Neuropsychological status (RBANS) in healthy South Africans showing that the MocA was fairly reliable but needed modification of certain domains items [53]. Specifically, it was suggested that in the absence of a culturally adapted version, lowering the cut-off to 24 reduces false positives [53]. The Kiswhali version of the MoCA, has been shown to be - linguistically and culturally adapted for evaluation of cognitive function in a rural Tanzanian population. Even so, the optimal cut-off with the best sensitivity and specificity was 19 [21]. Such an effort will equally be

Table 4

Comparison of neurocognitive test scores between PWE and Controls.

\begin{tabular}{|c|c|c|c|c|c|c|c|}
\hline \multirow[t]{2}{*}{ Neurocognitive test } & \multirow[t]{2}{*}{ Scores } & \multirow[t]{2}{*}{ Total } & \multicolumn{2}{|c|}{ PWE } & \multicolumn{2}{|c|}{ Controls } & \multirow[t]{2}{*}{ P-value } \\
\hline & & & $\mathrm{N}$ & $\%$ & $\mathrm{n}$ & $\%$ & \\
\hline Total & & 80 & 40 & 100.0 & 40 & 100.0 & \\
\hline \multirow[t]{2}{*}{ IHDS } & $<11$ & 49 & 33 & 82.5 & 16 & 40.0 & $<0.001$ \\
\hline & $\geq 11$ & 31 & 7 & 17.5 & 24 & 60.0 & \\
\hline \multirow[t]{2}{*}{ MOCA } & $<20$ & 50 & 35 & 87.5 & 15 & 37.5 & $<0.001$ \\
\hline & $\geq 20$ & 30 & 5 & 12.5 & 25 & 62.5 & \\
\hline \multirow[t]{2}{*}{ FAB } & $<13$ & 53 & 37 & 92.5 & 16 & 40.0 & $<0.001$ \\
\hline & $\geq 13$ & 27 & 3 & 7.5 & 24 & 60.0 & \\
\hline \multirow[t]{2}{*}{ Dubois } & $<9$ & 46 & 25 & 62.5 & 21 & 52.5 & 0.366 \\
\hline & $\geq 9$ & 34 & 15 & 37.5 & 19 & 47.5 & \\
\hline \multirow[t]{2}{*}{ Isaac } & $<30$ & 58 & 40 & 100.0 & 18 & 45.0 & $<0.001$ \\
\hline & $\geq 30$ & 22 & 0 & 0.0 & 22 & 55.0 & \\
\hline \multirow{2}{*}{ Clock test } & $<3$ & 12 & 3 & 7.5 & 9 & 22.5 & \\
\hline & $\geq 3$ & 68 & 37 & 92.5 & 31 & 77.5 & 0.060 \\
\hline
\end{tabular}

Table 5

Odd ratios for cognitive impairment in PWE and Controls.

\begin{tabular}{lllll}
\hline Neurocognitive test & Odds Ratio PWE:Controls & \multicolumn{2}{l}{$95 \% \mathrm{CI}$} & P-value \\
\cline { 3 - 4 } & & Lower & Upper & \\
\hline IHDS less than 11 & 7.07 & 2.29 & 23.19 & $<\mathbf{0 . 0 0 1}$ \\
MOCA less than 20 & 11.67 & 3.40 & 45.09 & $<\mathbf{0 . 0 0 1}$ \\
FAB less than 13 & 18.50 & 4.48 & 105.08 & $<\mathbf{0 . 0 0 1}$ \\
Dubois less than 9 & 1.51 & 0.56 & 4.05 & 0.498 \\
Isaac less than 30 & $\infty$ & 10.29 & $\infty$ & $<\mathbf{0 . 0 0 1}$ \\
CDT above 3 & 3.58 & 0.79 & 22.01 & 0.115 \\
\hline
\end{tabular}

necessary in our context. By applying standard cut-off of 26 in our study sample, there was no difference between cases and controls as up to $82,5 \%$ percent of the controls had poor results but the cut-off of 19 was more sensitive. Further research is however necessary to obtain a culturally adapted version of the MoCA and determine the best cut-off for cognitive impairment in our population. We have adapted a neuropsychological test battery and used it to develop norms for Cameroonian school-aged children in Yaoundé [54]. We have further used these normative data to evaluate neurocognitive impairment in children with sickle-cell disease [55]. However, this complete neuropsychological test battery could not be used in this study in the rural setting because of long administration time, its complexity and socio-cultural inadaptation. Developing neuropsychological tests to screen for cognitive impairment in people with epilepsy requires good understanding of cognitive domains that are most frequently affected in PWE [56]. In this study, PWE presented with significantly altered executive functions and verbal fluency compared to healthy subjects while memory and visuo-spatial functions were comparable in both groups. Several authors have reported executive dysfunction in patients with epilepsy and related to all epilepsy syndromes of childhood [57]. Executive function is dependent on the frontal lobe and its diverse connections to other parts of the cerebral cortex and the brain stem [57]. Thus a lesion in any part of the brain could result in executive dysfunction thus explaining why executive dysfunction can occur in all epilepsy syndromes both generalized and focal. Executive function is particularly important for educational achievement, employment, social function and a good quality of life [58]. The high prevalence of dysexecutive syndrome in our study sample could therefore contribute the high rate of school dropout observed in the epilepsy group. There is therefore a great need for special educational procedures in PWE particularly those with executive dysfunction [57].

Table 6

Neuropsychological parameters for Cases and Controls with Primary School level education only.

\begin{tabular}{llll}
\hline Variable & PWE + & PWE- & P-value \\
\hline Number & $\mathbf{3 6}$ & $\mathbf{2 1}$ & \\
Mean MoCA Score & $13.86 \pm 5.48$ & $19.10 \pm 5.54$ & $\mathbf{0 . 0 0 1}$ \\
Pathologic MoCA score (\%) & 97.2 & 90.5 & 0.271 \\
Mean IHDS score & $7.28 \pm 2.95$ & $9.43 \pm 2.46$ & $\mathbf{0 . 0 0 5}$ \\
Pathologic IHDS score (\%) & 86.1 & 52.4 & $\mathbf{0 . 0 0 5}$ \\
Mean FAB score & $6.92 \pm 3.64$ & $11.24 \pm 5.09$ & $\mathbf{0 . 0 0 2}$ \\
Pathologic FAB score (\%) & 100 & 76.2 & $\mathbf{0 . 0 0 2}$ \\
Mean learning score & $4.28 \pm 1.26$ & $4.62 \pm 1.12$ & 0.294 \\
Mean Memory score & $3.64 \pm 1.79$ & $3.43 \pm 1.96$ & 0.689 \\
Pathologic Dubois score (\%) & 66.7 & 57.1 & 0.472 \\
Mean clock test & $4.89 \pm 1.05$ & $4.43 \pm 1.50$ & 0.229 \\
Pathologic clock test (\%) & 97.1 & 90.5 & 0.283 \\
Mean Isaac's set test score & $14.71 \pm 5.13$ & $26.52 \pm 7.80$ & $<\mathbf{0 . 0 0 1}$ \\
Pathologic Isaac's set test (\%) & 100 & 61.9 & $<\mathbf{0 . 0 0 1}$ \\
\hline
\end{tabular}


Table 7

Factors independently associated with neurocognitive impairment.

\begin{tabular}{llllll}
\hline Score & Independently associated factor & OR & \multicolumn{2}{l}{$95 \% \mathrm{Cl}$} & \multirow{2}{*}{ P-value } \\
\cline { 4 - 5 } & & & Lower & Upper & \\
\hline \multirow{2}{*}{ IHDS } & Seizure Frequency & 2.252 & 0.983 & 5.156 & 0.055 \\
Isaac & Low level of education & 6.324 & 1.343 & 29.779 & 0.020 \\
& Length of stay $>15$ years in Bilomo & 1.094 & 1.019 & 1.173 & 0.013 \\
FAB & Low level of education & 8.96 & 2.14 & 37.522 & 0.003 \\
\hline
\end{tabular}

Deficits in verbal fluency have been described in various epileptic syndromes both generalized and focal $[59,60]$. Alterations in verbal fluency are a reflection of malfunctions in many other cognitive domains namely; language skills, semantic memory, executive functions (association between concepts and retrieval strategies) and psychomotor speed [61]. The relatively low level of education in this sample may have contributed to the impairment of verbal fluency. This is important as subjects perform poorer on verbal fluency tests when they are conducted in a language different from their mother tongue [62]. Assessing the participants in the Sanaga language would have probably yielded more precise results. This aspect should be taken into account in elaborating culturally adapted tools for neuropsychological evaluation in contexts such as ours.

Memory impairment is one of the first and most commonly affected cognitive domains in PWE $[13,63]$ but this was not demonstrated in this study. Other tests, based on the same paradigm of Five words of Dubois testing but with an increased number of words (either 16 or 48 ) could ameliorate the sensitivity and better detect memory issues in PWE [64]. Altered memory is more frequent in temporal lobe epilepsy where lesions of the hippocampus are frequent $[65,66]$. In our study population, there was a predominance of epilepsy with clinically reported generalized seizures. Few respondents reported symptoms compatible with temporal lobe epilepsy. However in the absence of EEG and imaging studies, this is difficult to ascertain. The absence of memory impairment might therefore be best explained by methodological issues. Some of the five words used in our sample, such as "Museum" and "Limonade" are quite unfamiliar in our population. Malian researchers proposed an adaptation of this cognitive test by not only translating the words in a local language but also choosing words among those commonly employed in the vocabulary of the general population. This modified version in Bambara proved to be more sensitive than the classical version of the Dubois' five-word testing [67].

Mean scores on clock testing and proportions with pathologic responses, were comparable in both groups. Clock testing is a measure of a number of cognitive functions: executive function, visuo-spatial ability and constructional praxia. The last two are more dependent on the integrity of the occipital and parietal lobes respectively. Visuospatial dysfunctions have been reported in children with occipital lobe epilepsy [68]. Occipital lobe epilepsies represent in certain series $8 \%$ of focal epilepsies according to the ILAE and could therefore explain the lower prevalence of visuospatial dysfunction compared to other cognitive deficits [69]. Very few respondents during this study reported any visual symptoms preceding or during the epileptic crises. Parietal lobe epilepsy is also quite rare representing about $6.3 \%$ of localization-related epilepsies in the same studies [69] However, the non-statistically significant difference in visuospatial function reported in our study might mainly be due to inadequacy of the clock test for rural populations with a low level of education. To the best of our knowledge, no previous study has used or validated the clock drawing test in rural African populations. Tanzanian researchers, in validating a Ki Swahili version of the MoCA, reported a high non completion rate on the clock drawing sub test in a Tanzanian rural population, probably due to the low level of education (particularly incomplete primary education) [21]. Our results support this hypothesis as more than $90 \%$ of participants, both cases and controls who had attended only primary school, had pathologic clock test scores.
We therefore posit that the cognitive domains affected in our study may be related to the underlying epilepsy syndrome as suggested by others [70]. Establishing a better correlation between the epilepsy syndromes and the neuropsychological profile requires EEG studies and occasionally, brain imaging which were not performed in this study for logistic reasons.

Previous research has established a strong relationship between level of education and cognitive performance [71]. Considering that the controls in our sample had a significantly higher level of education than the cases we analyzed the cognitive performance of two subgroups of cases and controls who had attended only primary education. The results were comparable to those obtained in the whole study population as a significant proportion of cases performed poorer on the IHDS, the FAB and the Isaac's set test. Though the mean MoCA scores were higher in the sub-group of PWE + with only primary education, the proportion of pathologic scores did not differ when considering the controls with the same level of education, suggesting rather the general need for adaptation of neuropsychological tests for this population. Borland et al. had earlier reported that a lower level of education was associated with lower scores on the MoCA in a large Swedish population-based cohort [72]. Nevertheless, this sub-group analysis strongly suggests that the lower level of education does not account for the higher level of global cognitive impairment as well as the dysexecutive syndrome and decreased verbal fluency in PWE compared to the controls. The duration of epilepsy correlated negatively with global cognitive performance. In active epilepsy, recurrent seizures lead to neuronal loss in encephalic regions that play a role in cognition (hippocampus, the frontal lobe, the thalamus and cerebellum) [73]. This mechanism could explain the relationship between frequency of seizures and cognitive impairment [74]. In this study PWE who had at least a seizure a week had an OR of 2.25 for global cognitive impairment. This implies that optimal management of the PWE so that they get into remission might be an important step towards decreasing the occurrence of cognitive deficits in this population.

Other factors not directly related to epilepsy equally contributed to specific cognitive dysfunction in this population. Participants who had attended only primary education had odds of 6.324 of having a pathologic score on the Isaac's set test and 8.96 for executive dysfunction. Education is considered to create a cognitive and a neurological reserve by increasing the efficacy of processing networks [75]. A recently published systematic review indicated that cognitive reserve and language processing capacities significantly increased in persons attending university classes compared with the control group who completed only the secondary school cycle. However, episodic memory, working memory and executive function did not differ significantly between groups [76]. The latter result on executive function contrasted with our finding of an association between low education cognitive dysfunction.

The length of stay in Bilomo village influenced the results on the Isaac's set Test. Those who had stayed there for more than 15 years had an OR of 1.094 for decreased verbal fluency. Interestingly, PWE stayed longer in the village compared to the controls. This could suggest a shared environmental risk factor for both epilepsy and cognitive impairment. We hypothesize that exposure to onchocerciasis which is common Bilomo could play a major role. Ninety-five per cent (95\%) of PWE in our sample met the criteria for onchocerciasis associated epilepsy. We had earlier reported an OV16 seropositivity rate of $46.9 \%$ in children in Bilomo indicating a high rate of ongoing onchocerciasis transmission [18]. Furthermore, hippocampal atrophy has been demonstrated in individuals infected with Onchocerca volvulus [77] suggesting a direct effect of the infection on cognition independent an associated epilepsy.

Future studies in this population may need to ascertain the extent to which onchocerciasis may affect the burden of cognitive impairment in people with onchocerciasis-associated epilepsy compared with people with epilepsy from other causes. Other factors may equally contribute 
to the high prevalence of neurocognitive impairment in our study population such as sleep disorders and psychiatric comorbidities particularly anxiety and depression all of which are habitual in epilepsy. This could be another path for further research [78-80].

This study has some major limitations. Most of the tests used to screen for cognitive impairment have not been cross-culturally adapted and validated for use in rural populations such as ours. This is particularly true for the MoCA, the Dubois' five-word testing and the clock test. Verbal fluency was assessed in French which is not the mother tongue of most of the participants. Consequently, differences in interpretation and significance of certain concepts may have resulted in an overestimation of cognitive dysfunction in this population. This is indicated by the high prevalence rates of cognitive impairment in the control population. Furthermore, most of the tests used in this study were screening tests and other cognitive functions such as psychomotor speed were not assessed. In the future, a more complete neuropsychological assessment battery may be necessary to adequately ascertain the burden of cognitive impairment in this population. This approach could be complemented by EEG, imaging studies and biological assays to diagnose onchocerciasis. This could yield interesting new data on the correlation between certain epilepsy syndromes and cognitive impairment in this population where most of the epilepsy appears to be attributable to onchocerciasis. Developing adapted tools to a rural population will be a next important step to have a more precise view of the magnitude of cognitive impairment in people living with epilepsy. These tests might need to be translated - in local languages and include concepts (both verbal and non-verbal) more familiar to rural populations. Large cohort studies will equally be important to quantify the role of onchocerciasis in the genesis of cognitive impairment in patients with OAE.

\section{Conclusion}

This study suggests that the prevalence of cognitive impairment might be much higher in PWE compared to the general population in the Mbam division particularly executive dysfunction and decreased verbal fluency. Longer disease duration and higher seizure frequency are associated with poorer cognitive performance. Our data suggest an environmental factor equally responsible for cognitive impairment possibly onchocerciasis infection. However the prevalence rates of cognitive impairment might be over-estimated in our sample hence the need for culturally adapted and appropriate tools for screening cognitive impairment in rural populations with epilepsy. Further research is necessary to establish the specific roles of epilepsy and onchocerciasis in the genesis of cognitive impairment in this population.

\section{Ethics statement}

Ethical clearance for the study was obtained from the Institutional Review Board of the Faculty of Medicine and Biomedical Sciences, of The University of Yaoundé I as well as the National Ethics Committee of Cameroon (Registration number 2017/02/875/CE/CNERSH/SP). As part of a larger ongoing study on the national territory, administrative authorization was obtained from the Ministry of Public Health of Cameroon (D30-177/L/MINSANTE/SG/DROS/TMC). Before field data collection, the administrative and health authorities of the Mbangassina sub-division were notified with all the study authorizations. Before inclusion, all participants signed a written informed consent form. All information obtained or used in the study was treated confidentially.

\section{Availability of data and material}

All collected data is confidentially kept at the Brain Research Africa Initiative (BRAIN), Cameroon. The datasets are available from the corresponding author on reasonable request.

\section{Declaration of Competing interests}

The authors declare no competing interests.

\section{Funding}

The study was supported by Brain Research Africa Initiative (BRAIN), www.brainafrica.org

\section{Authors' contributions}

AKN and JMA (Conception); AKN, SEC, SAA, ACZK, NR and JMA (Design); SEC, TGY, ENT, LN, MKM, DF, WYN, JBA, CN, ACZKB and AKN (Data collection); NLN, ENT, SEC, NR and AKN (Data analysis and interpretation); AKN and SEC wrote the first draft and all authors critically reviewed, and approved the final version of the manuscript.

\section{References}

[1] Fisher RS, WvE Boas, Blume W, Elger C, Genton P, Lee P, et al. Epileptic seizures and epilepsy: definitions proposed by the international league against epilepsy (ILAE) and the International Bureau for Epilepsy (IBE). Epilepsia. 2005;46(4):470-2.

[2] Merkena MD. Prevalence of cognitive adverse outcomes in epileptic outpatients. J Neurol Stroke. 2015;4(5):1-11.

[3] Arinzechi EO, Ogunrin OA, Nwosu CM, Nwani PO, Enwereji KO, Asomugha LA, et al. A community-based case-control study of prevalence and pattern of cognitive impairments in patients with epilepsy residing in south-eastern Nigeria. J Neurosci Rural Pract. 2016;7(3):405-11.

[4] Bugembe T. G309 epilepsy in Cameroon: a review of published literature for the development of a healthcare worker training tool. Arch Dis Child. 2014;99:A126 A7.

[5] Colebunders R, Njamnshi AK, van Oijen M, Mukendi D, Kashama JM, Mandro M, et al. Onchocerciasis-associated epilepsy: from recent epidemiological and clinical findings to policy implications. Epilepsia Open. 2017;2(2):145-52.

[6] Colebunders R, Mandro M, Njamnshi AK, Boussinesq M, Hotterbeekx A, Kamgno J, et al. Report of the first international workshop on onchocerciasis-associated epilepsy. Infect Dis Poverty. 2018;7(1):23.

[7] Winthrop KL, Furtado JM, Silva JC, Resnikoff S, Lansingh VC. River blindness: an old disease on the brink of elimination and control. J Glob Infect Dis. 2011;3(2):151-5.

[8] Chesnais CB, Nana-Djeunga HC, Njamnshi AK, Lenou-Nanga CG, Boullé C, Bissek A$\mathrm{CZ}-\mathrm{K}$, et al. The temporal relationship between onchocerciasis and epilepsy: a population-based cohort study. Lancet Infect Dis. 2018;18(11):1278-86.

[9] Chesnais CB, Bizet C, Campillo JT, Njamnshi WY, Bopda J, Nwane P, et al. A Second Population-Based Cohort Study in Cameroon Confirms the Temporal Relationship Between Onchocerciasis and Epilepsy. Open Forum Infectious Diseases. 2020;7(6).

[10] Diop AG, Hesdorffer DC, Logroscino G, Hauser WA. Epilepsy and mortality in Africa: a review of the literature. Epilepsia. 2005;46(s11):33-5.

[11] Johnson TP, Tyagi R, Lee PR, Lee MH, Johnson KR, Kowalak J, et al. Nodding syndrome may be an autoimmune reaction to the parasitic worm Onchocerca volvulus. Sci Transl Med. 2017;9(377).

[12] Kaddumukasa M, Kaddumukasa MN, Buwembo W, Munabi IG, Blixen C, Lhatoo S, et al. Epilepsy misconceptions and stigma reduction interventions in sub-Saharan Africa, a systematic review. Epilepsy \& behavior : E\&B. 2018;85:21-7.

[13] Witt JA, Helmstaedter C. Should cognition be screened in new-onset epilepsies? A study in 247 untreated patients. J Neurol. 2012;259(8):1727-31.

[14] Oyegbile TO, Dow C, Jones J, Bell B, Rutecki P, Sheth R, et al. The nature and course of neuropsychological morbidity in chronic temporal lobe epilepsy. Neurology. 2004; 62(10):1736-42.

[15] Helmstaedter C. THE IMPACT OF EPILEPSY ON COGNITIVE FUNCTION. Journal of Neurology, Neurosurgery \&amp; Psychiatry. 2013;84(9):e1 e.

[16] Berg AT. Epilepsy, cognition, and behavior: The clinical picture. Epilepsia. 2011;52 (Suppl. 1):7-12 Suppl 1.

[17] Njamnshi AKSV, VdP Djientcheu, Ongolo-Zogo P, Mapoure NY, Yepnjio FN, et al. Risk Factors Associated with Epilepsy in a Rural Area in Cameroon: A pilot study. Afr J of Neurol Sci. 2007;26(2):18-26.

[18] Siewe Fodjo JN, Tatah G, Tabah EN, Ngarka L, Nfor LN, Chokote SE, et al. Epidemiology of onchocerciasis-associated epilepsy in the Mbam and Sanaga river valleys of Cameroon: impact of more than 13 years of ivermectin. Infect Dis Poverty. 2018;7(1):114.

[19] Nasreddine ZS, Phillips NA, Bédirian V, Charbonneau S, Whitehead V, Collin I, et al. The Montreal cognitive assessment, MoCA: a brief screening tool for mild cognitive impairment. J Am Geriatr Soc. 2005;53(4):695-9.

[20] Phabphal K, Kanjanasatien J. Montreal cognitive assessment in cryptogenic epilepsy patients with normal mini-mental state examination scores. Epileptic disorders : international epilepsy journal with videotape. 2011;13(4):375-81.

[21] Masika GM, Yu DSF, Li PWC. Accuracy of the Montreal cognitive assessment in detecting mild cognitive impairment and dementia in the rural African population. $\mathrm{Ar}$ chives of clinical neuropsychology : the official journal of the National Academy of Neuropsychologists. 2020:acz086. https://doi.org/10.1093/arclin/acz086. 
[22] Pike NA, Poulsen MK, Woo MA. Validity of the Montreal cognitive assessment screener in adolescents and young adults with and without congenital heart disease. Nurs Res. 2017;66(3):222-30

[23] Sacktor NC, Wong M, Nakasujja N, Skolasky RL, Selnes OA, Musisi S, et al. The International HIV Dementia Scale: a new rapid screening test for HIV dementia. AIDS (London, England). 2005;19(13):1367-74

[24] Njamnshi AK, Djientcheu Vde P, Fonsah JY, Yepnjio FN, Njamnshi DM, Muna WE. The International HIV Dementia Scale is a useful screening tool for HIV-associated dementia/cognitive impairment in HIV-infected adults in Yaoundé-Cameroon. Journal of acquired immune deficiency syndromes (1999). 2008;49(4):393-7.

[25] B. Dubois JT, Portet F, Ousset PJ, Vellas B, Michel B. "Les 5 mots", épreuve simple et sensible pour le diagnostic de la maladie d'Alzheimer. 2002. . 2002;31:1696-9.

[26] Thomas-Anterion C, Laurent B. Les marqueurs neuropsychologiques du diagnostic de la maladie d'Alzheimer. Rev Neurol. 2006;162(10):913-20.

[27] Bitonto LDDC, Cretin B. L'épiLepsie du Lobe temporaLà La consuLtation mémoire: Vérifier la plainte Le problème des outils d'évaluation. Neurologies. 2013;16(157): $138-42$.

[28] Guerchet M, Houinato D, Paraiso MN, von Ahsen N, Nubukpo P, Otto M, et al. Cognitive impairment and dementia in elderly people living in rural Benin, West Africa. Dement Geriatr Cogn Disord. 2009;27(1):34-41.

[29] Guerchet M, M'Belesso P, Mouanga AM, Bandzouzi B, Tabo A. Houinato DS, et al. Prevalence of dementia in elderly living in two cities of Central Africa: the EDAC survey. Dement Geriatr Cogn Disord. 2010;30(3):261-8.

[30] Bouattour NFN, Hadjkacem H, Hdiji O, Sakka S, Dammak M, Mhiri C. Etalonnage du test des cinq mots dans une population tunisienne de sujets sains. Pan Afr Med J. 2019;34(58).

[31] Dubois B, Slachevsky A, Litvan I, Pillon B. The FAB: a frontal assessment battery at bedside. Neurology. 2000;55(11):1621-6.

[32] Beato R, Amaral-Carvalho V, Guimarães HC, Tumas V, Souza CP. Oliveira GNd, et al. frontal assessment battery in a Brazilian sample of healthy controls: normative data. Arq Neuropsiquiatr. 2012;70:278-80.

[33] Agah E, Asgari-Rad N, Ahmadi M, Tafakhori A, Aghamollaii V. Evaluating executive function in patients with temporal lobe epilepsy using the frontal assessment battery. Epilepsy Res. 2017;133:22-7.

[34] Rasaholiarison NFRR, Rajaonarison LA, Rakotomanana JL Razafimahefa J, Tehindrazanarivelo AD. Fréquence et caractéristiques des AVC impliquant les artères perforantes dans le Service de Neurologie de l'Hopital Bafelatanana. Antananarivo Pan African Medical Journal. 2017;28:17.

[35] Cunha PJ, PAd Oliveira, Cortezzi M, Busatto GF, Scivoletto S. Executive dysfunction and low academic attainment in adolescent substance abusers with a history of maltreatment. MedicalExpress. 2015;2.

[36] Croisile B. Comment examiner la mémoire en pratique médicale courante ? Pratique Neurologique - FMC. 2012;3:32-9.

[37] Isaacs B, Kennie AT. The set test as an aid to the detection of dementia in old people. The British journal of psychiatry : the journal of mental science. 1973;123(575): 467-70.

[38] Corne H, Sylvestre G, Magnin E, Vieillard S, Portha C, Chopard G, et al. Intérêt du bilan neuropsychologique en centre périphérique, dans le cadre des bilans préchirurgicaux chez des patients souffrant d'épilepsie du lobe temporal : une analyse multicas. Rev Neuropsychol. 2012;4(4):231-41.

[39] Mendez MF, Ala T, Underwood KL. Development of scoring criteria for the clock drawing task in Alzheimer's disease. J Am Geriatr Soc. 1992;40(11):1095-9.

[40] Yaksa BKK, Harahap HS, Amalia E, Indrayana Y. The effect of the onset of seizure on clock drawing test score of epilepsy patients. . 2018;2018:7.

[41] Santana I, Duro D, Freitas S, Alves L, Simões M. The clock drawing test: Portuguese norms, by age and education, for three different scoring systems. Archives of clinical neuropsychology : the official journal of the National Academy of Neuropsychologists. 2013;28

[42] Ghanizadeh A, Safavi S, Berk M. Clock face drawing test performance in children with ADHD. Basic and clinical neuroscience. 2013;4(1):50-6.

[43] Fisher RS, Cross JH, French JA, Higurashi N, Hirsch E, Jansen FE, et al. Operational classification of seizure types by the international league against epilepsy: position paper of the ILAE Commission for Classification and Terminology. Epilepsia. 2017; 58(4):522-30.

[44] Siewe JFN, Ngarka L, Tatah G, Mengnjo MK, Nfor LN, Chokote ES, et al. Clinical presentations of onchocerciasis-associated epilepsy (OAE) in Cameroon. Epilepsy Behav. 2019;90:70-8.

[45] Epilepsy CoCaTotILA. Proposal for revised classification of epilepsies and epileptic syndromes. Commission on classification and terminology of the international league against epilepsy. Epilepsia. 1989;30(4):389-99.

[46] Colebunders R, Nelson Siewe FJ, Hotterbeekx A. Onchocerciasis-associated epilepsy, an additional reason for strengthening Onchocerciasis elimination programs. Trends Parasitol. 2018;34(3):208-16.

[47] Mavrodaris A, Powell J, Thorogood M. Prevalences of dementia and cognitive impairment among older people in sub-Saharan Africa: a systematic review. Bull World Health Organ. 2013;91(10):773-83.

[48] Huang CW, Hsieh YJ, Tsai JJ, Pai MC. Cognitive performance in cryptogenic epilepsy. Acta Neurol Scand. 2005;112(4):228-33.

[49] Siewe Fodjo JN, Mandro M, Mukendi D, Tepage F, Menon S, Nakato S, et al. Onchocerciasis-associated epilepsy in the Democratic Republic of Congo: clinical description and relationship with microfilarial density. PLoS Negl Trop Dis. 2019;13 (7): $\mathrm{e} 0007300$

[50] Vogt VL, Äikiä M, Del Barrio A, Boon P, Borbély C, Bran E, et al. Current standards of neuropsychological assessment in epilepsy surgery centers across Europe. Epilepsia. 2017;58(3):343-55.
[51] Yu J, Li J, Huang X. The Beijing version of the Montreal cognitive assessment as a brief screening tool for mild cognitive impairment: a community-based study. BMC Psychiatry. 2012;12:156

[52] Robbins RN, Joska JA, Thomas KGF, Stein DJ, Linda T, Mellins CA, et al. Exploring the utility of the Montreal cognitive assessment to detect HIV-associated neurocognitive disorder: the challenge and need for culturally valid screening tests in South Africa. Clin Neuropsychol. 2013;27(3):437-54.

[53] Beath N, Asmal L, van den Heuvel L, Seedat S. Validation of the Montreal cognitive assessment against the RBANS in a healthy south African cohort. The South African journal of psychiatry : SAJP : the journal of the Society of Psychiatrists of South Africa. 2018:24:1304

[54] Ruffieux N, Njamnshi A, Mayer E, Sztajzel R, Eta S, Doh R, et al. Neuropsychology in Cameroon: first normative data for cognitive tests among school-aged children. Child neuropsychology : a journal on normal and abnormal development in childhood and adolescence. 2009;16:1-19.

[55] Ruffieux N, Njamnshi AK, Wonkam A, Hauert CA, Chanal J, Verdon V, et al. Association between biological markers of sickle cell disease and cognitive functioning amongst Cameroonian children. Child Neuropsychol. 2013;19(2):143-60.

[56] Kurzbuch K, Pauli E, Gaál L, Kerling F, Kasper BS, Stefan H, et al. Computerized cognitive testing in epilepsy (CCTE): a new method for cognitive screening. Seizure. 2013;22(6):424-32.

[57] Høie B, Mykletun A, Waaler PE, Skeidsvoll H, Sommerfelt K. Executive functions and seizure-related factors in children with epilepsy in western Norway. Developmental Medicine \& Child Neurology. 2006;48(6):519-25.

[58] Moffitt TE, Arseneault L, Belsky D, Dickson N, Hancox RJ, Harrington H, et al. A gradient of childhood self-control predicts health, wealth, and public safety. Proc Natl Acad Sci. 2011;108(7):2693-8.

[59] Witt JA, Helmstaedter C. Cognition in the early stages of adult epilepsy. Seizure. 2015;26:65-8.

[60] Metternich B, Buschmann F, Wagner K, Schulze-Bonhage A, Kriston L. Verbal fluency in focal epilepsy: a systematic review and meta-analysis. Neuropsychol Rev. 2014; 24.

[61] You SJ. Cognitive function of idiopathic childhood epilepsy. Korean J Pediatr. 2012; 55(5):159-63.

[62] Narme P, Maillet D, Palisson J, Le Clésiau H, Moroni C, Belin C. How to assess executive functions in a low-educated and multicultural population using a switching verbal fluency test (the TFA-93) in neurodegenerative diseases? American Journal of Alzheimer's Disease \& Other Dementiasr. 2019;34(7-8):469-77.

[63] Mathuranath PS, George A, Cherian PJ, Alexander A, Sarma SG, Sarma PS. Effects of age, education and gender on verbal fluency. J Clin Exp Neuropsychol. 2003;25(8): 1057-64.

[64] Dupont MP, Pelix C, Toulza O, Trivalle C, Baud M, Jacus JP. Intérêts et limites de la procédure de Grober et Buschke dans le diagnostic précoce de la maladie d'Alzheimer et des démences mixtes. NPG Neurologie - Psychiatrie - Gériatrie. 2008;8(44):25-34.

[65] Butler CR, Zeman AZ. Recent insights into the impairment of memory in epilepsy: transient epileptic amnesia, accelerated long-term forgetting and remote memory impairment. Brain : a journal of neurology. 2008;131(Pt 9):2243-63.

[66] Hermann B, Seidenberg M. Epilepsy and cognition. Epilepsy Curr. 2007;7(1):1-6.

[67] Guinto CO, Coulibaly T, Koné Z, Coulibaly S, Maiga B, Dembélé K, et al. Socio-cultural adaptation and standardization of Dubois' five words testing in a population of normal subject in Mali. West Africa eNeurologicalSci. 2016;3:60-3.

[68] Santangelo G, Trojano L, Vitale C, Improta I, Alineri I, Meo R, et al. Cognitive dysfunctions in occipital lobe epilepsy compared to temporal lobe epilepsy. J Neuropsychol. 2017;11(2):277-90.

[69] Sveinbjornsdottir S, Duncan JS. Parietal and occipital lobe epilepsy: a review. Epilepsia. 1993;34(3):493-521.

[70] MacAllister WS, Vasserman M, Rosenthal J, Sherman E. Attention and executive functions in children with epilepsy: what, why, and what to do. Applied neuropsychology Child. 2014;3(3):215-25.

[71] Guerra-Carrillo B, Katovich K, Bunge SA. Does higher education hone cognitive functioning and learning efficacy? Findings from a large and diverse sample. PLoS One. 2017;12(8):e0182276.

[72] Borland E, Nägga K, Nilsson PM, Minthon L, Nilsson ED, Palmqvist S. The Montreal cognitive assessment: normative data from a large Swedish population-based cohort. Journal of Alzheimer's disease : JAD. 2017;59(3):893-901.

[73] Rudzinski LA, Meador KJ. Epilepsy and neuropsychological comorbidities. Continuum (Minneapolis, Minn). 2013;19(3 Epilepsy):682-96.

[74] Vingerhoets G. Cognitive effects of seizures. Seizure. 2006;15(4):221-6.

[75] Vadikolias K, Tsiakiri-Vatamidis A, Tripsianis G, Tsivgoulis G, Ioannidis P, Serdari A, et al. Mild cognitive impairment: effect of education on the verbal and nonverbal tasks performance decline. Brain Behav. 2012;2(5):620-7.

[76] Matyas N, Keser Aschenberger F, Wagner G, Teufer B, Auer S, Gisinger C, et al. Continuing education for the prevention of mild cognitive impairment and Alzheimer'stype dementia: a systematic review and overview of systematic reviews. BMJ Open. 2019;9(7):e027719.

[77] Hotterbeekx A, Namale Ssonko V, Oyet W, Lakwo T, Idro R. Neurological manifestations in Onchocerca volvulus infection: a review. Brain Res Bull. 2019:145:39-44.

[78] Mazarati A. Epilepsy and forgetfulness: one impairment. Multiple Mechanisms Epilepsy Curr. 2008;8(1):25-6.

[79] Bazil CW. Epilepsy and sleep disturbance. Epilepsy \& behavior : E\&B. 2003;4(Suppl. 2):S39-45.

[80] Chang HJ, Liao CC, Hu CJ, Shen WW, Chen TL. Psychiatric disorders after epilepsy diagnosis: a population-based retrospective cohort study. PLoS One. 2013;8(4): e59999. 